\title{
X-ray accretion signatures in the close CTTS binary V4046 Sagittarii
}

\author{
H. M. Günther ${ }^{1}$, C. Liefke ${ }^{1}$, J. H. M. M. Schmitt ${ }^{1}$, J. Robrade ${ }^{1}$, and J.-U. Ness ${ }^{2}$ \\ 1 Hamburger Sternwarte, Gojenbergsweg 112, 21029 Hamburg, Germany \\ e-mail: moritz.guenther@hs.uni-hamburg.de \\ 2 Arizona State University, School of Earth and Space Exploration, Tempe, AZ, 85287-1404, USA
}

Received 28 August 2006 / Accepted 26 September 2006

ABSTRACT

\begin{abstract}
We present Chandra HETGS observations of the classical T Tauri star (CTTS) V4046 Sgr. The He-like triplets of O VII, Ne IX, and Si XIII are clearly detected. Similar to the CTTS TW Hya and BP Tau, the forbidden lines of O VII and Ne IX are weak compared to the intercombination line, indicating high plasma densities in the X-ray emitting regions. The Si XIII triplet, however, is within the low-density limit, in agreement with the predictions of the accretion funnel infall model with an additional stellar corona. V4046 Sgr is the first close binary exhibiting these features. Together with previous high-resolution X-ray data on TW Hya and BP Tau, and in contrast to T Tau, now three out of four CTTS show evidence of accretion funnels.
\end{abstract}

Key words. X-rays: stars - stars: individual: V4046 Sagittarii - stars: pre-main sequence - stars: coronae - stars: activity

\section{Introduction}

T Tauri stars are young (age $<10 \mathrm{Myr})$, low-mass $\left(M_{*}<3 M_{\odot}\right)$ stars evolving down to the main sequence in the HertzsprungRussell diagram; a detailed review of pre-main sequence stellar evolution is given by Feigelson \& Montmerle (1999). The CTTS subclass shows signs of active accretion from a circumstellar disk as proven by strong infrared excess and a large $\mathrm{H} \alpha$ equivalent width $(>10 \AA)$. Current models of CTTS assume a dipolar stellar magnetic field, disrupting the disk near the corotation radius, where ionised material is loaded onto magnetic field lines and is accelerated to essentially free-fall velocity along a magnetic funnel (Shu et al. 1994). A shock is formed close to the stellar surface and the material passing through the shock is heated up to temperatures in the MK range. The accretion geometries for binaries are understood less well theoretically, but see Artymowicz \& Lubow (1996).

The X-ray emission from TTS was first detected with the Einstein Observatory, and extensive X-ray studies of a variety of star forming regions were undertaken with ROSAT. However, to what extent the observed X-ray emission can actually be attributed to accretion processes remained unclear and is the subject of some debate, since a central result of stellar X-ray astronomy states that all "cool stars", i.e., stars with outer convective envelopes, are surrounded by hot coronae (Schmitt \& Liefke 2004). The usual interpretation of this finding is that turbulence in the outer convective zone leads to the production of magnetic fields and the ensuing activity, making it natural to attribute the observed X-ray emission from CTTS to the phenomena of magnetic activity rather than of accretion.

Imaging studies with low/medium spectral resolution allow large samples of TTS to be studied simultaneously as was recently performed for the Orion nebula by the COUP project (Feigelson 2005). In such studies, stars with and without accretion disks are difficult to distinguish; however, statistical studies seem to indicate some differences between CTTS and those TTS without accreting disks (Stelzer et al. 2000; Stelzer \& Neuhäuser 2001; Flaccomio et al. 2003).

Only with the high spectral resolution of grating spectra, as available for a few individual CTTS observed with Chandra and $X M M-N e w t o n$, is it possible to identify spectroscopic signatures taht distinguish accretion and coronal emission and to reliably derive the physical properties of the emission region through temperature, density, and abundance diagnostics. Specifically, a high spectral resolution survey of 48 stellar coronae by Ness et al. (2004) showed that most of the coronal densities are compatible with the low-density limit within the error ranges.

Only two clear exceptions among cool stars are known to date, the CTTS TW Hya and BP Tau. In the Chandra HETGS spectrum of TW Hya, Kastner et al. (2002) found unusually high densities and interpreted them as the signature of an accretion funnel; this was then confirmed by an XMM-Newton observation described by Stelzer \& Schmitt (2004). The same low f/i-ratios for the O VII and Ne IX triplets were observed in XMM-Newton RGS spectra of the CTTS BP Tau (Schmitt et al. 2005), while the XMM-Newton EPIC spectra also clearly show the presence of rather hot plasma. The authors interpret the BP Tau data in terms of accretion with an additional active corona; similar conclusions were drawn by Robrade \& Schmitt (2006) in a comparative study of the high and medium resolution X-ray data of four CTTS. The interpretation of the $\mathrm{f} / \mathrm{i}$-ratios in terms of density is not unambiguous. Ness \& Schmitt (2005) presented a careful examination of density-sensitive iron lines in TW Hya and also showed that neither the UV flux nor line scattering affects the He-like triplets.

In this letter we present Chandra HETG spectra of the CTTS V4046 Sgr, which we show to closely resemble the X-ray spectra of TW Hya and BP Tau and to be the third star with very low 
Total ACIS-S lightcurve: V4046 Sgr (ObsIDs 5423 \& 6265)

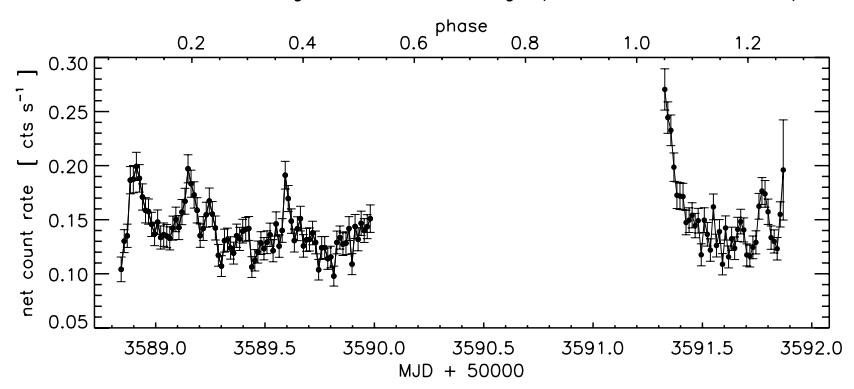

Fig. 1. X-ray lightcurve of V4046 Sgr, extracted from the 0th order data and dispersed spectra. The upper axis shows the orbital phase using the ephemerides from Stempels \& Gahm (2004).

He-like f/i-ratios. We therefore suggest that accretion-related Xray emission may in fact be quite common in CTTS.

\section{V4046 Sgr: observations and data reduction}

V4046 Sgr is a nearby CTTS at a distance between $42 \mathrm{pc}$ (Hutchinson et al. 1990) and 83 pc (Quast et al. 2000). The star is isolated from any dark or molecular cloud and has negligible extinction. The CTTS nature of V4046 Sgr has been established through its variable $\mathrm{H} \alpha$ equivalent width (30-120 $\AA$, Byrne 1986) and a strong infrared excess, IRAS-detected (de La Reza et al. 1986), while other IR observations are also consistent with a disk surrounding V4046 Sgr (Hutchinson et al. 1990). V4046 Sgr itself is a spectroscopic binary with a welldetermined orbital period of 2.4213459 days (Stempels \& Gahm 2004), consisting of a K7 V and a K5 V star with masses $M_{1}=$ $0.86 M_{\odot}$ and $M_{2}=0.69 M_{\odot}$ at an orbital separation of less than $10 R_{\odot}$ (Quast et al. 2000). The observed spectral energy IR distribution is consistent with an inner disk radius of $1.8 \mathrm{AU}$ (Jensen \& Mathieu 1997), the disk surrounding V4046 Sgr is thus circumbinary (Quast et al. 2000). The viewing angle of the system is $i=35-45^{\circ}$, and the symmetry axis of both the disk and the binary are supposed to coincide. The high-resolution optical spectroscopy of higher Balmer lines indicates gas clouds associated with the stars well within the inner radius of the circumbinary disk (Stempels \& Gahm 2004).

V4046 Sgr was observed with the Chandra HETGS in two blocks, starting on 6 Aug. 2006 for $100 \mathrm{ks}$ (ObsID 5423) and on 9 Aug. 2006 for 50 ks (ObsID 6265). First-order grating spectra were extracted by applying standard CIAO 3.3 tools, and then positive and negative orders were summed up. Individual emission lines in the HEG and MEG spectra were analysed with the CORA line-fitting tool (Ness \& Wichmann 2002), assuming modified Lorentzian line profiles with $\beta=2.5$.

\section{Results}

The X-ray lightcurve of V4046 Sgr is shown in Fig. 1. Several phases of enhanced flux levels are apparent, where the hardness of the X-ray radiation increases marginally; the second observation starts with what looks like the decay phase of a large flare. Variations in the shape of the higher Balmer line profiles are known to correlate with the orbital phase (Stempels \& Gahm 2004), but we do not find any obvious correlation between X-ray flux and phase. We analysed single and combined spectra and found no significant differences between the two observations; therefore, we use the merged spectra in all of our analysis.
Table 1. Measured line fluxes for V4046 Sgr.

\begin{tabular}{lrrrr}
\hline \hline Line ID & $\begin{array}{r}\lambda \\
(\AA)\end{array}$ & $\begin{array}{r}\text { MEG } \\
\text { (counts) }\end{array}$ & $\begin{array}{r}\text { HEG } \\
\text { (counts) }\end{array}$ & $\begin{array}{r}\text { Photon } \\
\text { flux }^{a}\end{array}$ \\
\hline Si XIV Ly $\alpha$ & 6.18 & $32 \pm 6$ & $15 \pm 4$ & $2.5 \pm 0.7$ \\
Si XIII r & 6.64 & $46 \pm 8$ & $15 \pm 4$ & $2.7 \pm 0.5$ \\
Si XIII i & 6.69 & $10^{b}$ & $9 \pm 3$ & $1.5 \pm 0.5$ \\
Si XIII f & 6.74 & $36 \pm 7$ & $20 \pm 5$ & $2.9 \pm 0.7$ \\
Mg XII Ly $\alpha$ & 8.42 & $29 \pm 6$ & n.a. & $1.6 \pm 0.4$ \\
Mg XI r & 9.17 & $35 \pm 7$ & n.a. & $2.7 \pm 0.5$ \\
Ne X Ly $\beta$ & 10.23 & $57 \pm 8$ & n.a. & $5.2 \pm 0.7$ \\
Ne X Ly $\alpha$ & 12.14 & $280 \pm 17$ & $102 \pm 10$ & $48.7 \pm 3.0$ \\
Ne IX r & 13.46 & $245 \pm 16$ & $64 \pm 8$ & $74.2 \pm 4.8$ \\
Ne IX i & 13.56 & $107 \pm 11$ & $26 \pm 5$ & $32.0 \pm 3.3$ \\
Ne IX f & 13.70 & $105 \pm 11$ & $23 \pm 5$ & $33.2 \pm 3.3$ \\
O VIII Ly $\beta$ & 16.02 & $23 \pm 5$ & n.a. & $11.0 \pm 2.0$ \\
O VIII Ly $\alpha$ & 18.97 & $98 \pm 10$ & n.a. & $115.7 \pm 11.8$ \\
O VII r & 21.6 & $21 \pm 5$ & n.a. & $49.3 \pm 11.7$ \\
O VII i & 21.8 & $10.4 \pm 3.5$ & n.a. & $26.8 \pm 9.0$ \\
O VII f & 22.1 & $3.0 \pm 2.2$ & n.a. & $9.0 \pm 7.0$ \\
N VII Ly $\alpha$ & 24.78 & $28 \pm 6$ & n.a. & $75.0 \pm 16.1$ \\
Fe XVII & 15.01 & $38 \pm 7$ & n.a. & $17.0 \pm 3.2$ \\
Fe XVII & 15.26 & $21 \pm 5$ & n.a. & $8.1 \pm 1.9$ \\
Fe XVII & 17.05 & $21 \pm 5$ & n.a. & $14.0 \pm 0.3$ \\
Fe XVII & 17.10 & $22 \pm 5$ & n.a. & $14.6 \pm 0.3$ \\
\hline
\end{tabular}

${ }^{a}$ In units of $10^{-6}$ photons $\mathrm{cm}^{-2} \mathrm{~s}^{-1}$ calculated from the MEG data except for the Si XIII triplet, where only an upper limit is available for the Si XIII i line; ${ }^{b} 2 \sigma$ upper limit.

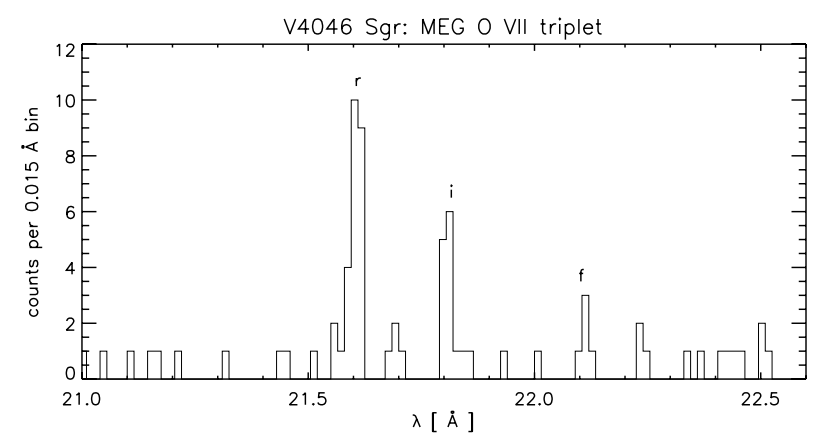

Fig. 2. The V4046 Sgr MEG count spectrum around the O VII triplet region.

The MEG and HEG grating spectra show a typical emissionline spectrum. Specifically, the Ly $\alpha$ lines of silicon, magnesium, neon, oxygen, and nitrogen and the Ly $\beta$ lines of neon and oxygen are detected (cf. Fig. 5). Weak iron lines are found from Fe XVII and Fe XVIII; He-like resonance lines are detected from oxygen, neon, and silicon, while the He-like $\mathrm{Mg}$ lines are very weak. The forbidden and intercombination lines of oxygen, neon, and silicon are clearly detected and resolved. The strongest detected lines, together with the derived best-fit line counts and their errors (as determined with CORA), are listed in Table 1.

In Fig. 2 we plot the observed MEG count spectrum in the O VII triplet region around $22 \AA$. Obviously, the signal is low, and yet the $r$ and $i$ lines are clearly detected with extremely high significance (cf. Table 1). However, only $3.0 \pm 2.2$ counts are recorded from the $f$ line, clearly indicating deviations from the low-density limit. We specifically find $\mathrm{f} / \mathrm{i}=0.33 \pm 0.28$ for $\mathrm{O}$ VII, which must be compared to the low-density limit of 3.9 (Smith et al. 2001). Figure 3 shows the observed MEG count spectrum in the Ne IX triplet region around $13.5 \AA$. All triplet lines are clearly detected and, as shown by an inspection of the MEG spectrum, contamination by Fe XIX (cf. Ness et al. 2003) is of the 


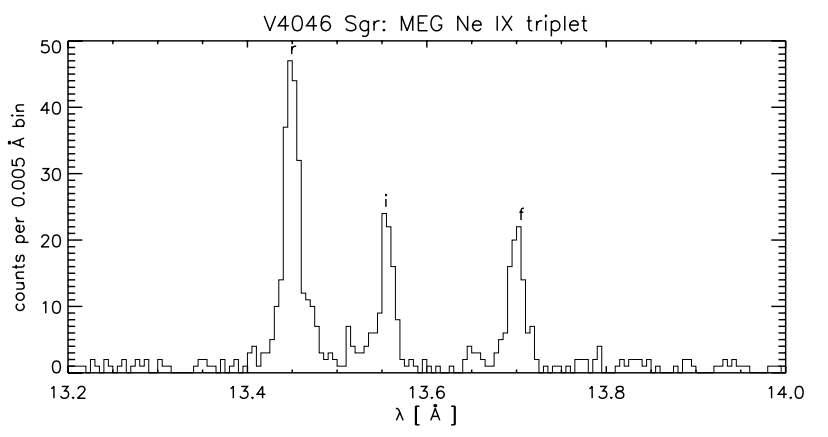

Fig. 3. The V4046 Sgr MEG count spectrum around the Ne IX triplet region.

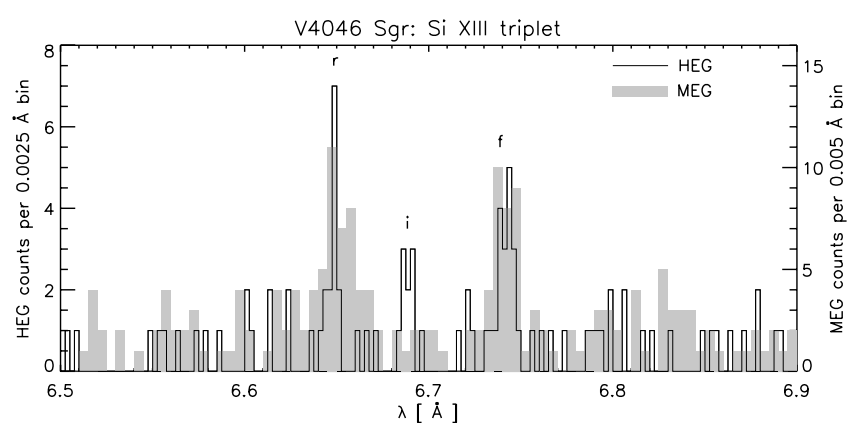

Fig. 4. Si XIII triplet region of V4046 Sgr in the HEG (black line) and MEG (shaded). The intercombination line is clearly stronger in the HEG than in the MEG, but the fits in Table 1 show that both measurements are statistically consistent.

order of a few percent at its worst for V4046 Sgr similar to the case of TW Hya (Stelzer \& Schmitt 2004). The Ne IX f/i-ratio is $1.04 \pm 0.15$, derived under the assumption of negligible iron contamination, with the Ne IX low-density limit being 3.1. Finally, in Fig. 4 we plot the observed HEG and MEG count spectrum in the Si XIII triplet region around $6.7 \AA$.

All triplet lines are clearly detected in the HEG spectrum, the $\mathrm{f}$ line being approximately at the same strength as the $r$ line. While only an upper limit for the i line can be obtained from the MEG spectrum, a Si XIII f/i-ratio of $1.9 \pm 0.6$ can be derived from the HEG spectrum, which compares well with the Si XIII f/iratios of the larger sample of active stars studied by Testa et al. (2004). Since the Si XIII f line is located exactly on the Si K edge of the detector, we investigated whether the observed Si XIII f/iratio (in recorded counts) is consistent with the $\mathrm{Si}$ XIII $\mathrm{f} / \mathrm{i}$-count ratio for a larger sample of stars. Assuming then that all active stars are in the low-density limit for Si XIII with $\log n_{\mathrm{e}}<13.5$, we conclude that the same applies for V4046 Sgr (as far as Si XIII is concerned). This conclusion is supported by the ratio of Fe XVII 17.10 ̊/17.05 ̊ of about unity, which Ness \& Schmitt (2005) argue to be the low-density limit $\left(\log n_{\mathrm{e}}<13.6\right)$; however, uncertain atomic physics require some caution.

\section{Discussion}

The X-ray data on the CTTS TW Hya and BP Tau have been interpreted in terms of an accretion funnel scenario, where the $\mathrm{X}$-ray emission is emitted in a shock ("hot spot") produced by the infall of material along the magnetic field essentially at freefall velocity onto the stellar surface. The main reason for this interpretation was the anomalously low O VII f/i-ratio observed for TW Hya and BP Tau, since the extensive spectral survey by Ness et al. (2004) found no star that would even come close to
Table 2. Comparison of properties of the three CTTS TW Hya, BP Tau, and V4046 Sgr.

\begin{tabular}{lcccc}
\hline \hline & TW Hya & BP Tau & V4046 Sgr \\
\hline \multicolumn{5}{c}{ f/i ratios } \\
Si XIII & n.a & n.a. & $1.9 \pm 0.6$ & $<13.5$ \\
Ne IX & $0.33 \pm 0.24$ & $0.40 \pm 0.26$ & $1.05 \pm 0.15$ & 12.0 \\
O VII & $0.05 \pm 0.05$ & $0.37 \pm 0.16$ & $0.33 \pm 0.28$ & 11.5 \\
\hline \multicolumn{5}{c}{ Ly $\alpha / r$ ratios } \\
$\mathrm{Si}$ & $1.83 \pm 0.61$ & n.a. & $1.13 \pm 0.27$ & $7.10 \pm 0.05$ \\
$\mathrm{Mg}$ & $0.60 \pm 0.30$ & n.a. & $1.03 \pm 0.30$ & $6.91 \pm 0.05$ \\
$\mathrm{Ne}$ & $0.63 \pm 0.07$ & $2.51 \pm 0.85$ & $0.70 \pm 0.09$ & $6.61 \pm 0.01$ \\
$\mathrm{O}$ & $2.02 \pm 0.41$ & $1.59 \pm 0.35$ & $2.36 \pm 0.60$ & $6.51 \pm 0.05$ \\
\hline \multicolumn{5}{c}{ abundance ratios } \\
$A_{\mathrm{Si}} / A_{\mathrm{Mg}}$ & $1.63 \pm 0.68$ & n.a. & $1.58 \pm 0.36$ & 0.93 \\
$A_{\mathrm{Mg}} / A_{\mathrm{Ne}}$ & $0.05 \pm 0.02$ & n.a. & $0.08 \pm 0.02$ & 0.32 \\
$A_{\mathrm{Ne}} / A_{\mathrm{O}}$ & $0.86 \pm 0.11$ & $0.66 \pm 0.20$ & $1.04 \pm 0.14$ & 0.18 \\
\hline
\end{tabular}

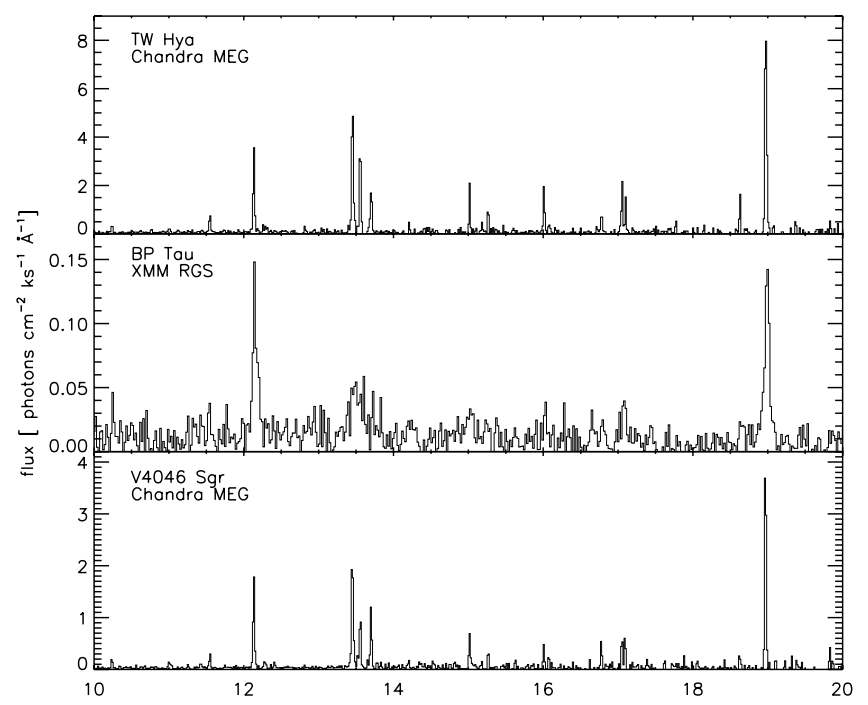

Fig. 5. Comparison of the X-ray flux spectra of TW Hya, BP Tau, and V4046 Sgr.

the low O VII f/i-ratio observed for those two stars. The question is therefore, wether the very same scenario also applies to the CTTS V4046 Sgr and possibly to CTTS as a class?

In Fig. 5 we compare the X-ray spectra in the range $10 \AA$ to $20 \AA$ for the three TW Hya (MEG), BP Tau (XMM RGS), and CTTS V4046 Sgr (MEG). Clearly, the energy resolution of the RGS instrument and the SNR of the BP Tau data set is lower than that of the MEG spectra. Nevertheless, some common trends, as well as differences, appear: In all three cases, the O VIII Ly $\alpha$ line is the strongest line, and neon lines are stronger than iron lines, which are weak if not absent. In BP Tau the Ne X Ly $\alpha$ line is much stronger than the Ne IX He-like $r$ line, the opposite applies to TW Hya and V4046 Sgr. The different Ly $\alpha$ to He-like resonance line ratios for our three CTTS are summarised in Table 2. Interpreting these ratios in terms of an effective temperature, we note that the emission measure in V4046 Sgr cannot be described by a single temperature emission model. We also investigated the Ly $\alpha /$ Ly $\beta$ ratios for Ne X and O VIII and compared the temperatures obtained in this way to estimates from the ratio of Ly $\alpha$ to He-like resonance line. The temperatures found in this way are both about 0.1 dex below the peak formation temperatures of their respective ionisation stages. We find that the ratios are fully consistent with optically thin emission, even if the absorption to 
V4046 Sgr is as large as $N_{\mathrm{H}}=10^{21} \mathrm{~cm}^{-2}$. A second tracer of resonant scattering is the ratio Fe XVII 15.26 $\mathrm{A} / 15.01 \AA$, which matches optically thin predictions as well.

With regard to the He-like f/i-ratios for TW Hya, BP Tau, and V4046 Sgr (cf. Table 2), we find the observed O VII f/i-ratio of V4046 Sgr to compare well with what is observed for BP Tau and to be fully consistent with the lower value of TW Hya. When interpreted as a pure density diagnostics (i.e., assuming no contaminating UV flux), we find $\log n_{\mathrm{e}} \approx 11.5$ (Smith et al. 2001). Thus TW Hya, BP Tau, and V4046 Sgr are in marked contrast to the CTTS T Tau, where Güdel et al. (2006) measured an f/i ratio of $\sim 4$ and inferred an upper limit to the electron density of $1.4 \times 10^{10} \mathrm{~cm}^{-3}$ (68\% confidence). Ne IX is formed at higher temperatures, and the Ne IX f/i-ratio of V4046 Sgr is somewhat higher than those measured for TW Hya and BP Tau, but still smaller than all the $48 \mathrm{Ne}$ IX f/i-ratios determined by Ness et al. (2004) for their sample of active stars. The formal Ne IX density derived for V4046 Sgr is $\log n_{\mathrm{e}} \approx 12.0$. We stress that the $\mathrm{f} / \mathrm{i}$ ratios for both Ne IX and O VII (peak formation temperatures $\log T \approx 6.6$ and $\log T \approx 6.3$, respectively) clearly deviate from the low-density limit; hence, the X-ray flux is unlikely to be produced in a stellar corona. On the contrary, the f/i-ratio measured for the Si XIII triplet agrees with the Si XIII f/i-ratios found in the survey by Testa et al. (2004); the Si XIII peak formation temperature of $\log T \approx 7.0$ cannot be reached in an accretion shock, so this triplet probably has a coronal origin.

Turning now to elemental abundances we use ratios of linear combinations of the measured fluxes of the H-like Ly $\alpha$ and He-like resonance lines to derive abundance ratios that are, as a first approximation, independent of the underlying temperature structure of the emitting plasma (Liefke \& Schmitt 2006). In Table 2 we list the values of $A_{\mathrm{Si}} / A_{\mathrm{Mg}}, A_{\mathrm{Mg}} / A_{\mathrm{Ne}}$, and $A_{\mathrm{Ne}} / A_{\mathrm{O}}$ obtained for the three CTTS, as well as the solar value (Grevesse \& Sauval 1998) for comparison. Drake et al. (2005) found the $\mathrm{Ne} / \mathrm{O}$ abundance in BP Tau consistent with their sample of postT Tauri stars, while Stelzer \& Schmitt (2004) and Schmitt et al. (2005) interpret the - compared to solar - enhanced Ne/O abundances as due to grain depletion. Also, V4046 Sgr shows the same enhanced $\mathrm{Ne} / \mathrm{O}$ abundances as found for TW Hya and BP Tau. As a consequence, the $\mathrm{Mg} / \mathrm{Ne}$ abundance is low, both for V4046 Sgr and for TW Hya; however, the relative Si/Mg abundances are comparable to solar values.

\section{Conclusions}

For single CTTS, the model of an accreting hot spot, where material impacts onto the stellar surface at free-fall velocity, closely matches the observational data of both TW Hya (Kastner et al. 2002; Stelzer \& Schmitt 2004) and BP Tau (Schmitt et al. 2005). Detailed simulations (Calvet \& Gullbring 1998; Lamzin 1998; Günther et al. 2006) predict the emissivity, the line ratios, the UV field, and the veiling continuum. V4046 Sgr fits fully in this picture. Optically thin emission from an accretion hot spot explains the unusually low f/i-ratios observed in O VII and Ne IX. A "normal" corona accounts for the more energetic $\mathrm{X}$-rays and, in particular, for the low-density Si XIII triplet, as well as for the variability of V4046 Sgr. This is somewhat surprising since the accretion geometry of V4046 Sgr is far more complicated because of its binary nature. Gas clouds close to the stars can be traced by line profile analysis of higher Balmer lines and seem to exist in the vicinity of the stars, yet no circumstellar disk should exist due to the small binary separation.
The variability of the X-ray lightcurve suggests energetic events as observed for the case of BP Tau, as well as in the coronae of other stars; and we caution that densities of up to a few $10^{11} \mathrm{~cm}^{-3}$ (Güdel et al. 2002) seem to be occasionally reached in flares. As to abundances, V4046 Sgr shows the very high Ne/O abundance, reminiscent of TW Hya, and grain depletion is a suggestive scenario to explain the apparently high $\mathrm{Ne}$ abundance.

In summary, three of four CTTS with available highresolution and high SNR X-ray grating spectra show far lower f/i ratios in the O VII and Ne IX triplets than any other star. In V4046 Sgr we are likely to see the emission from a corona in the high-temperature Si lines, and the binary nature of V4046 Sgr seems to have little influence on its X-ray spectrum. We thus conclude that the presence, not the absence, of low f/i ratios appears to be more typical of CTTS. Also, the emission mechanism obviously works in single and close binary stars, presenting a challenge for future magnetohydrodynamic simulations of the accretion funnel.

Acknowledgements. H.M.G., C.L., and J.R. acknowledge support from the DLR under grant 50OR0105. J.-U.N. gratefully acknowledges support provided by NASA through Chandra Postdoctoral Fellowship grant PF5-60039 awarded by the Chandra X-ray Center, which is operated by the Smithsonian Astrophysical Observatory for NASA under contract NAS8-03060.

\section{References}

Artymowicz, P., \& Lubow, S. H. 1996, ApJ, 467, L77

Byrne, P. B. 1986, Ir. Astron. J., 17, 294

Calvet, N., \& Gullbring, E. 1998, ApJ, 509, 802

de La Reza, R., Quast, G., Torres, C. A. O., et al. 1986, in New Insights in Astrophysics, Eight Years of UV Astronomy with IUE, ed. E. J. Rolfe, ESA SP-263, 107

Drake, J. J., Testa, P., \& Hartmann, L. 2005, ApJ, 627, L149

Feigelson, E. 2005, in Star Formation in the Era of Three Great Observatories Feigelson, E. D., \& Montmerle, T. 1999, ARA\&A, 37, 363

Flaccomio, E., Micela, G., \& Sciortino, S. 2003, A\&A, 402, 277

Grevesse, N., \& Sauval, A. J. 1998, Space Sci. Rev., 85, 161

Güdel, M., Audard, M., Skinner, S. L., \& Horvath, M. I. 2002, ApJ, 580, L73

Güdel, M., Skinner, S. L., Mel'nikov, S.-Y., et al. 2006, A\&A, submitted

Günther, H. M., Schmitt, J. H. M. M., Robrade, J., \& Liefke, C. 2006, A\&A, submitted

Hutchinson, M. G., Evans, A., Winkler, H., \& Spencer Jones, J. 1990, A\&A, 234, 230

Jensen, E. L. N., \& Mathieu, R. D. 1997, AJ, 114, 301

Kastner, J. H., Huenemoerder, D. P., Schulz, N. S., Canizares, C. R., \& Weintraub, D. A. 2002, ApJ, 567, 434

Lamzin, S. A. 1998, Astron. Rep., 42, 322

Liefke, C., \& Schmitt, J. H. M. M. 2006, A\&A, accepted

Ness, J.-U., Brickhouse, N. S., Drake, J. J., \& Huenemoerder, D. P. 2003, ApJ, 598,1277

Ness, J.-U., Güdel, M., Schmitt, J. H. M. M., Audard, M., \& Telleschi, A. 2004, A\&A, 427, 667

Ness, J.-U., \& Schmitt, J. H. M. M. 2005, A\&A, 444, L41

Ness, J.-U., \& Wichmann, R. 2002, Astron. Nachr., 323, 129

Quast, G. R., Torres, C. A. O., de La Reza, R., da Silva, L., \& Mayor, M. 2000, in IAU Symp., 28P

Robrade, J., \& Schmitt, J. H. M. M. 2006, A\&A, 449, 737

Schmitt, J. H. M. M., \& Liefke, C. 2004, A\&A, 417, 651

Schmitt, J. H. M. M., Robrade, J., Ness, J.-U., Favata, F., \& Stelzer, B. 2005, A\&A, 432, L35

Shu, F., Najita, J., Ostriker, E., et al. 1994, ApJ, 429, 781

Smith, R. K., Brickhouse, N. S., Liedahl, D. A., \& Raymond, J. C. 2001, ApJ, 556, L91

Stelzer, B., \& Neuhäuser, R. 2001, A\&A, 377, 538

Stelzer, B., Neuhäuser, R., \& Hambaryan, V. 2000, A\&A, 356, 949

Stelzer, B., \& Schmitt, J. H. M. M. 2004, A\&A, 418, 687

Stempels, H. C., \& Gahm, G. F. 2004, A\&A, 421, 1159

Testa, P., Drake, J. J., \& Peres, G. 2004, ApJ, 617, 508 